\title{
A High-Isolation Dual-Polarization Substrate-Integrated Fabry-Pérot Cavity Antenna
}

\author{
Chang Chen, Bo-Liang Liu, Ling Ji, and Wei-Dong Chen \\ Key Laboratory of Electromagnetic Space Information, Chinese Academy of Sciences, \\ University of Science and Technology of China, Hefei 230027, China \\ Correspondence should be addressed to Chang Chen; chench@ustc.edu.cn
}

Received 27 June 2014; Revised 23 October 2014; Accepted 14 November 2014

Academic Editor: Giuseppe Mazzarella

Copyright (C) 2015 Chang Chen et al. This is an open access article distributed under the Creative Commons Attribution License, which permits unrestricted use, distribution, and reproduction in any medium, provided the original work is properly cited.

A dual-polarization substrate-integrated Fabry-Pérot cavity (SI-FPC) antenna is presented in this paper. The patch embedded in SI-FPC is excited with a near-field coupled feeding structure for V-polarization and with a slot-coupled feeding structure for Hpolarization. The feeding structures are separated by a ground plane to improve the isolation between the ports. As a design example, an antenna operating at $10.0 \mathrm{GHz}$ is fabricated and measured. A high degree of port isolation $(<-40 \mathrm{~dB})$ over the whole operating bandwidth $(9.5-10.2 \mathrm{GHz})$ and good cross-polarization level $(>25 \mathrm{~dB})$ can be achieved.

\section{Introduction}

Dual-polarized antennas have been widely used in wireless communication systems to suppress multipath propagation effects and reduce installation costs $[1,2]$. Such antennas are usually designed as arrays with high-gain property to execute long distance transmission with a lower power. In [2], a $2 \times 4$ electromagnetically coupled patch array is well designed, but the circuit losses and mutual coupling bring the effective array gain from $16.2 \mathrm{dBi}$ to $12.7 \mathrm{dBi}$. It is desirable to seek singleaperture structure with high gain to eliminate the feed loss and complexity.

Fabry-Pérot cavity (FPC) antenna is used to satisfy high gain requirement in a limited aperture [3]. Former studies have designed dual-band air-filled FPC antennas with two orthogonal linear polarizations $[4,5]$. However, the stiffness of air-filled cavity structure cannot be guaranteed. What is more, the minimum height of air-filled FPC is $\lambda_{0} / 2$ due to the resonant condition.

Fully substrate-integrated FPC (SI-FPC) $[6,7]$ is presented to realize a high-gain antenna with lower profile and better mechanical robustness. In [8], subwavelength SI-FPC applies the AMC sheet as a partially reflective surface. The profile of the antenna is kept at only $\lambda_{0} / 9$ and the realized gain can still achieve $12 \mathrm{dBi}$. To the best of our knowledge, there is no dual-polarization antenna based on SI-FPC technology reported in the literature.

In this paper, we present an SI-FPC antenna for realization of a high-gain dual-polarization antenna with high isolation due to the separation of the feeding structures. The primary radiation patch embedded in the cavity is fed by a smaller patch with a two-branch feeding strip for vertical polarization and by a microstrip line under a metallic ground plane through a crossed slot for horizontal polarization. The prototype operating at $10.0 \mathrm{GHz}$ is designed with fullwave simulation and validated experimentally. The proposed antenna is compared to previous works $[4,5]$ to evaluate its performance.

As a result, the proposed antenna successfully achieves high isolation, high gain, and broader bandwidth with a simple structure.

\section{Antenna Design}

To design a proper FPC for the proposed antenna, the configurations of PRS and cavity are designed first. Using a ray optics model for infinite PRS [9], the directivity is attained by multiplying wave reflections inside the cavity. For the FP 


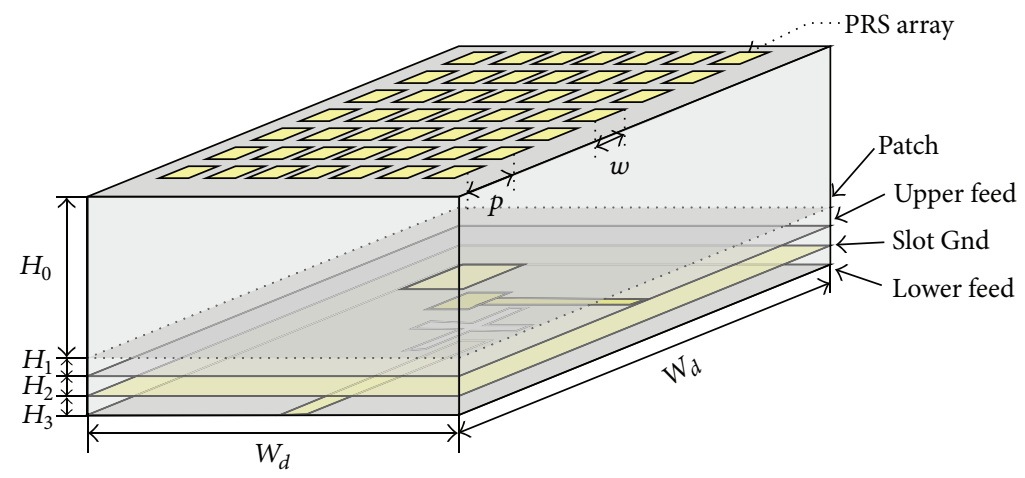

FIGURE 1: The prototype of the SI-FPC antenna.

cavity fully filled with air between the PRS and ground, the resonance condition is

$$
-\pi+\varphi_{\mathrm{prs}}-\frac{4 \pi h}{\lambda_{0}}=2 N \pi, \quad N=0, \pm 1, \pm 2
$$

where $\varphi_{\text {prs }}$ and $-\pi$ are the reflection phase of PRS and ground plane, respectively, and $h$ indicates the thickness of the FPC. If PRS is made of a single layer of metallic patches, with the reflection phase close to $\pi$, the lowest cavity height is about $\lambda_{0} / 2$.

Figure 1 shows the proposed antenna comprised of a partial reflective surface (PRS) and a dual-polarization patch embedded at the center of Fabry-Pérot cavity (FPC) with detailed dimensions in Table 1 . The cavity is fully integrated by multilayered dielectric boards (Rogers RT5880, $\varepsilon_{r}=2.2$, $\tan \delta=0.0009$ ) for low antenna profile and easy fabrication compared to air-filled FPC. The overall dimension of the antenna is $63.4 \times 63.4 \times 12.1 \mathrm{~mm}$ (or $\left.2.1 \times 2.1 \times 0.4 \lambda_{0}\right)$. The height of the FPC antenna is about $20 \%$ lower than the airfilled FPC design.

The PRS is mounted on the top substrate and is actually formed by a $7 \times 7$ metallic square patch array as shown in Figure 1. The reflective phase of PRS is determined by the unit cell period $p$ and the size of square patch $w$. On the top of the second board a $9 \times 9 \mathrm{~mm}^{2}$ patch radiator operating at $10 \mathrm{GHz}$ is embedded. The dual-polarized feeding structure of the patch radiator is built on the two bottom substrates and excites two orthogonal modes of TE10 and TE01 in the patch.

To achieve a high isolation in the whole operating band, the electric fields of dual polarizations are excited symmetrically to keep them strictly orthogonal and the strong coupling between two input lines in the cavity is eliminated. Therefore, the radiation patch is fed by two symmetrical feeding structures printed on different layers and separated by a metallic ground plane with a crossed slot as shown in Figure 2. Compared to the former dual-polarization antennas [10], this design can eliminate the use of an air bridge for bridging two input lines on the same layer. Hence, the isolation is improved and the structure is easier to fabricate. For the upper layer, it consists of a smaller patch whose length is $X_{2}$ and a two-branch feeding strip at the edge of the patch.
TABLE 1: Antenna parameters (unit: millimeter).

\begin{tabular}{lccccc}
\hline Parameter & Value & Parameter & Value & Parameter & Value \\
\hline$H_{0}$ & 10.5 & $X_{1}$ & 9 & $L_{S}$ & 7 \\
$H_{1}$ & 0.508 & $X_{2}$ & 8 & $L_{1}$ & 1.2 \\
$H_{2}$ & 0.508 & $W_{1}$ & 0.6 & $L_{2}$ & 9 \\
$H_{3}$ & 0.508 & $W_{2}$ & 1.2 & $L_{3}$ & 1.4 \\
$p$ & 9 & $W_{3}$ & 7 & $L_{4}$ & 5.5 \\
$w$ & 8.4 & $W_{4}$ & 1.5 & $L_{5}$ & 25 \\
$W_{d}$ & 63.4 & $W_{S}$ & 0.5 & & \\
\hline
\end{tabular}

The smaller patch excites the radiating patch through nearfield coupling and the two-branch feeding strip structure is introduced for higher polarized isolation.

For the lower layer, a fork-like feedline under the ground plane excites the radiation patch through a centered crossed slot and a high degree of geometrical and electrical symmetry is achieved. By exciting the patch symmetrically, the electric fields induced through the slot are with the same direction and with similar amplitude. As a consequence, the linear polarization of $\mathrm{H}$-polarization port is more prominent along $y$-direction. The purity of the linear polarization can be verified by the distribution of surface currents on the patch which is shown in Figure 3. It is shown that the two linear polarizations are strictly orthogonal and the current at Portl excited by Port 2 is extremely small and vice versa. A high isolation $(<-40 \mathrm{~dB})$ between two ports over the entire operating band is achieved.

\section{Results and Discussions}

The antenna was fabricated, as shown in Figure 4. The cavity is substrate-integrated by a multilayered dielectric board (Rogers RT5880, $\varepsilon_{r}=2.2, \tan \delta=0.0009$ ) with the overall dimension of $63.4 \mathrm{~mm} \times 63.4 \mathrm{~mm} \times 12.1 \mathrm{~mm}(2.1 \times 2.1 \times$ $\left.0.4 \lambda_{0}\right)$. A $7 \times 7$ square patch array is etched on the top surfaces of a $3.125 \mathrm{~mm}$ thick Rogers RT5880 substrate. A total of seven Rogers RT5880 substrates are assembled together to form the proposed antenna. Since a thick substrate with the height of about $10.5 \mathrm{~mm}$ is difficult to manufacture and costly, the gap of FPC between radiator patch and PRS is filled with 


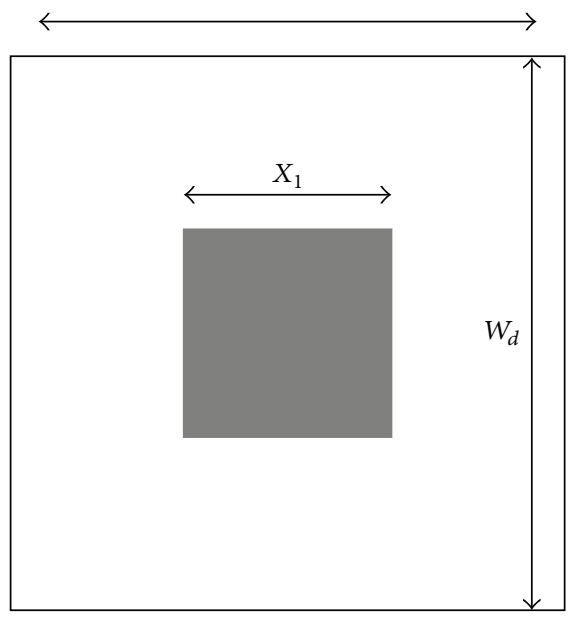

(a)

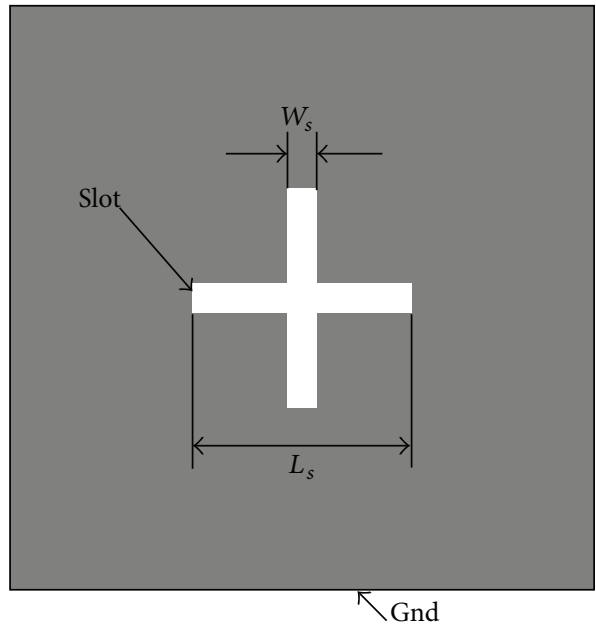

(c)

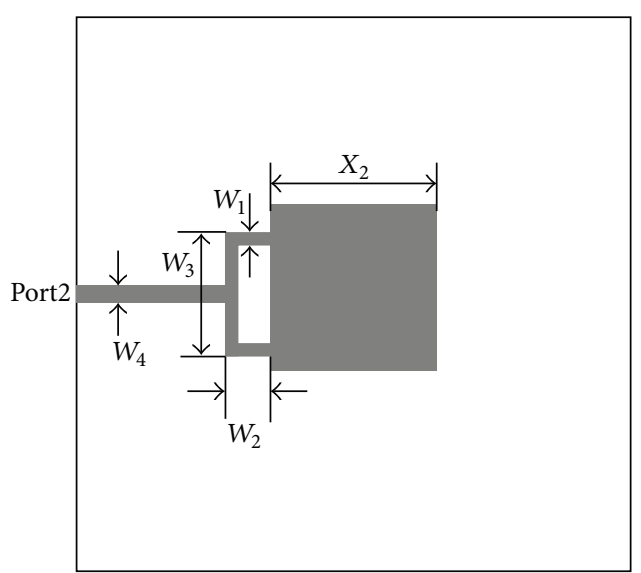

(b)

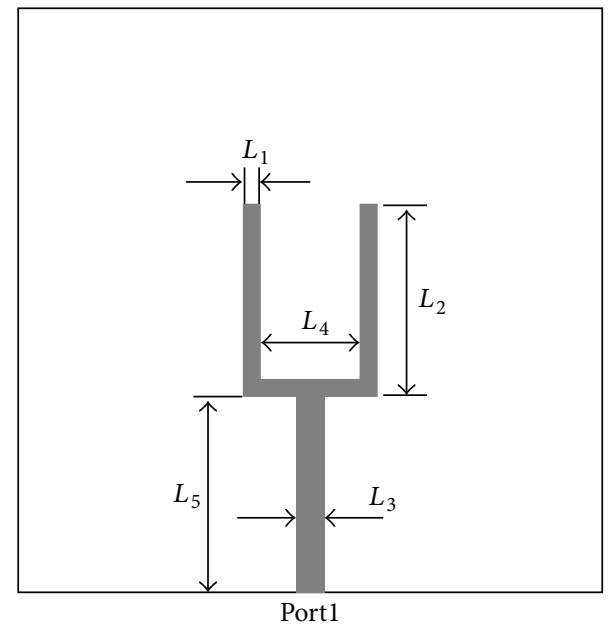

(d)

FIgURE 2: The feeding structure of the SI-FPC antenna: (a) patch radiator, (b) upper feed, (c) slot ground plane, and (d) lower feed.

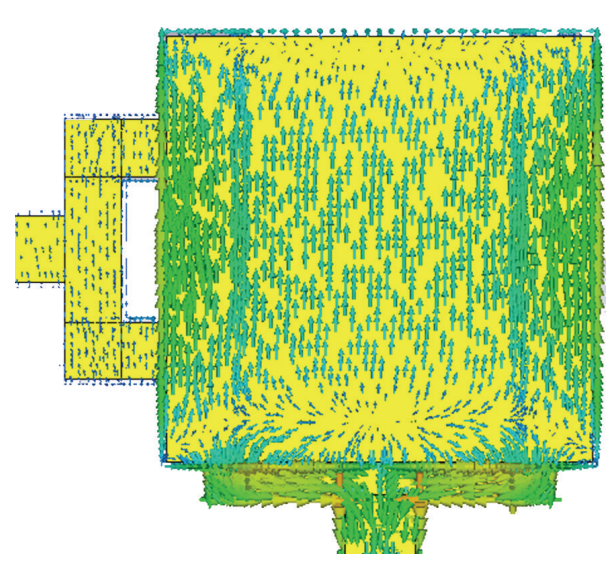

(a)
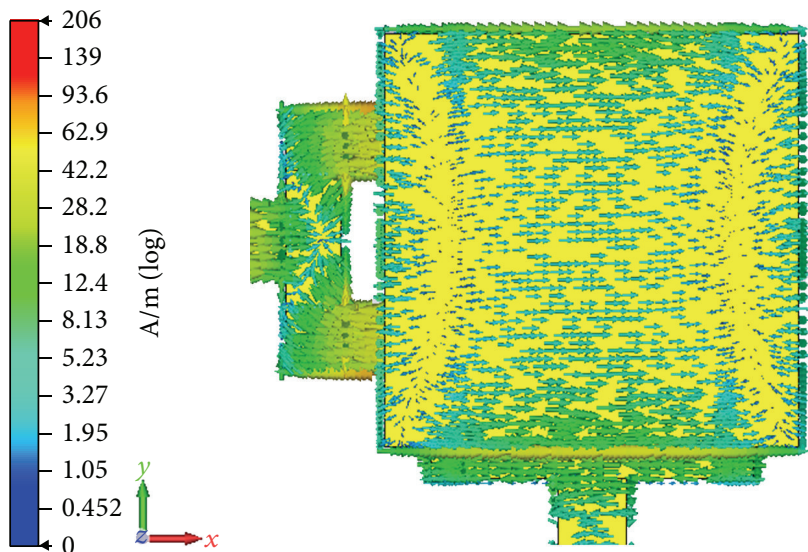

(b)

FIGURE 3: Surface current distributions on the patch radiator: (a) vertical polarization and (b) horizontal polarization. 
TABLE 2: Comparison among the proposed antenna and former results.

\begin{tabular}{|c|c|c|c|c|c|c|}
\hline & Frequency/GHz & Size $/ \lambda_{0}$ & Isolation/dB & Cross-polarization/dB & $\mathrm{BW} / \%$ & Gain/dBi \\
\hline This work & 10.00 & $2.1 \times 2.1 \times 0.4$ & $<-40$ & -25 & 7.1 & 15.8 \\
\hline$[4]$ & 10.25 & $5.4 \times 5.4 \times 0.5$ & $<-22$ & $-30 /-27$ & 4.9 & 18.0 \\
\hline$[5]$ & 5.20 & $3.0 \times 3.0 \times 0.4$ & N/A & $-30 /-21$ & 5.1 & 14.5 \\
\hline
\end{tabular}
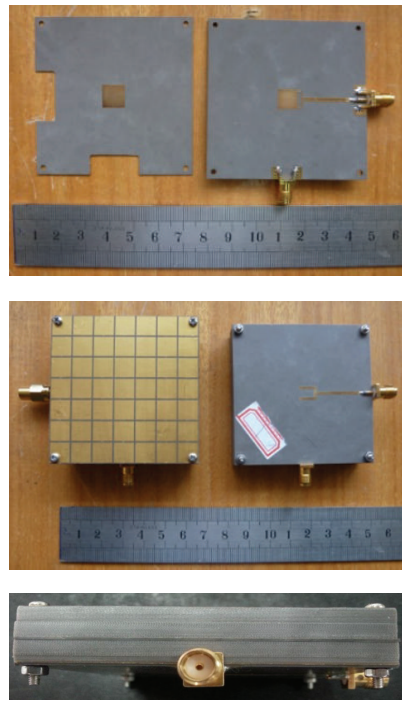

Figure 4: The photograph of the fabrication SI-FPC antenna.

four separate substrates, three of which are $3.125 \mathrm{~mm}$ and one is $1.13 \mathrm{~mm}$ high. All of the substrates are tightened with each other by glues and bolts. The stiffness of the antenna is experimentally validated.

In Figure 5, the measured impedance bandwidth is shown larger than $8.3 \%(9.44-10.26 \mathrm{GHz})$ for both ports. The resonant frequency shift of $2 \%$ between measured and simulated results is due to the fabrication tolerance. Moreover, the simulated and measured isolation between two ports is more than $40 \mathrm{~dB}$ throughout the operating bandwidth. The difference between measured and simulated isolation after $9.9 \mathrm{GHz}$ is due to the very low measured signal level at this region, for which the VNA might not be accurate enough to measure signals at such a level $(<-50 \mathrm{~dB})$.

Figure 6 presents the measured gain of both polarizations. The maximum gain is $15.5 \mathrm{dBi}$ at $10.0 \mathrm{GHz}$ for $\mathrm{V}$-polarization (fed by Portl) and $14.9 \mathrm{dBi}$ at $9.8 \mathrm{GHz}$ for $\mathrm{H}$-polarization (fed by Port2). Compared to simulated results, the gain loss of $1 \mathrm{~dB}$ is caused by the air-gap and displacement of dielectric sheets during the prototype assembling. The measured $3 \mathrm{~dB}$ gain bandwidth of realized gain is about $7.1 \%(9.5-10.2 \mathrm{GHz})$. Therefore, the common bandwidth is about $7.1 \%$ based on measured results.

Figure 7 presents the measured E-plane and $\mathrm{H}$-plane radiation patterns, $\mathrm{V}$-polarization and $\mathrm{H}$-polarization at $9.8 \mathrm{GHz}$, which are the frequencies of the maximum gain. The crosspolarization level is better than $-25 \mathrm{~dB}$ within measured $3 \mathrm{~dB}$ beamwidth of $24.1^{\circ}$ for E-plane and $21.4^{\circ}$ for $\mathrm{H}$-plane. The side

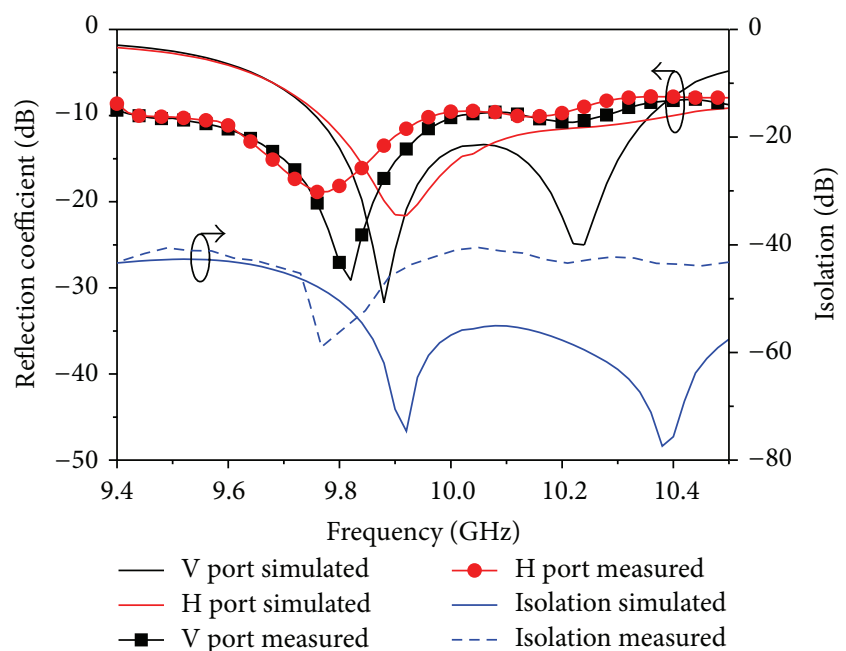

FIGURE 5: Measured and simulated return loss and isolation of the prototype SI-FPC antenna.

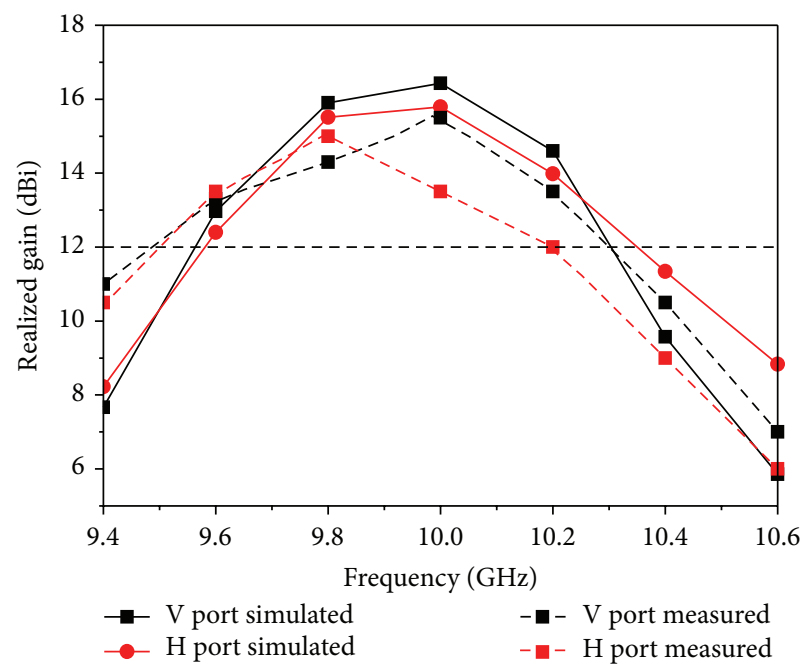

FIGURE 6: Measured and simulated realized gain of the prototype SI-FPC antenna.

lobe level is about $-10 \mathrm{~dB}$ because of undesired surface wave at the edges of the cavity. The front-to-back ratio of the antenna is about $15 \mathrm{~dB}$.

To evaluate the performance, Table 2 shows the comparison between the proposed antenna and previous works $[4,5]$. The proposed antenna is substrate-integrated and operates at a single frequency for both polarizations, while the exhibiting antenna designs are air-filled and operate 


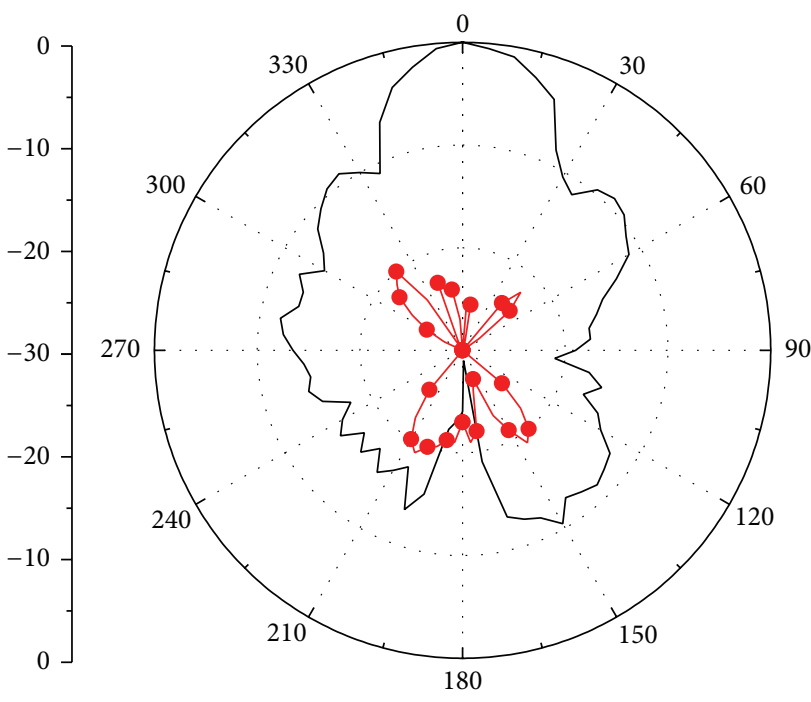

(a)

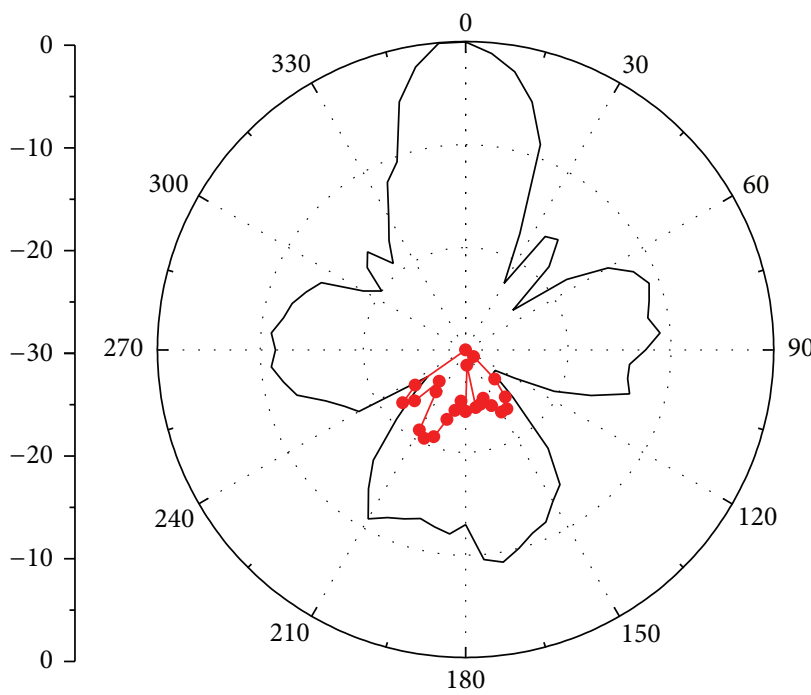

- Copolar, E-plane

$\rightarrow$ Cross-polar, E-plane

(c)

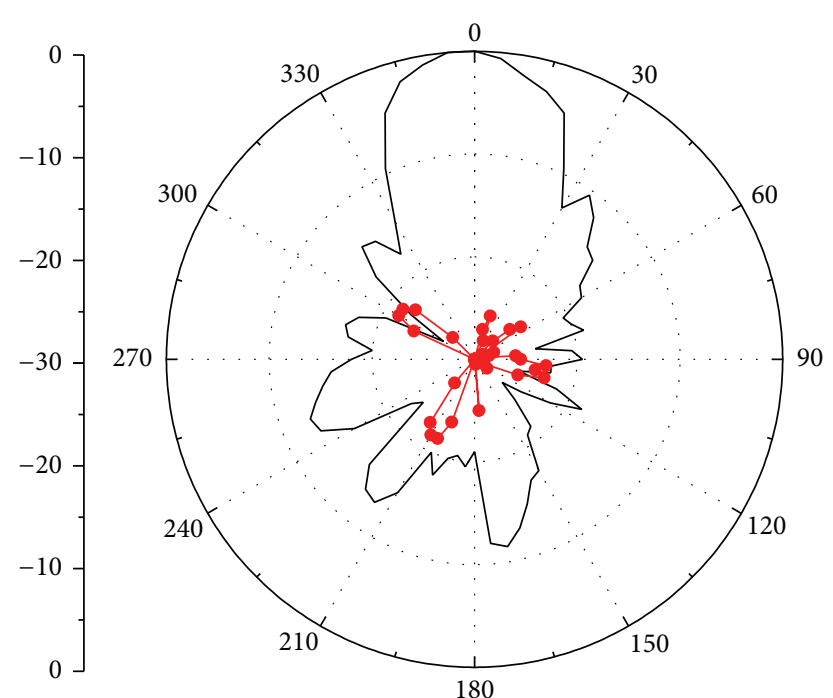

(b)

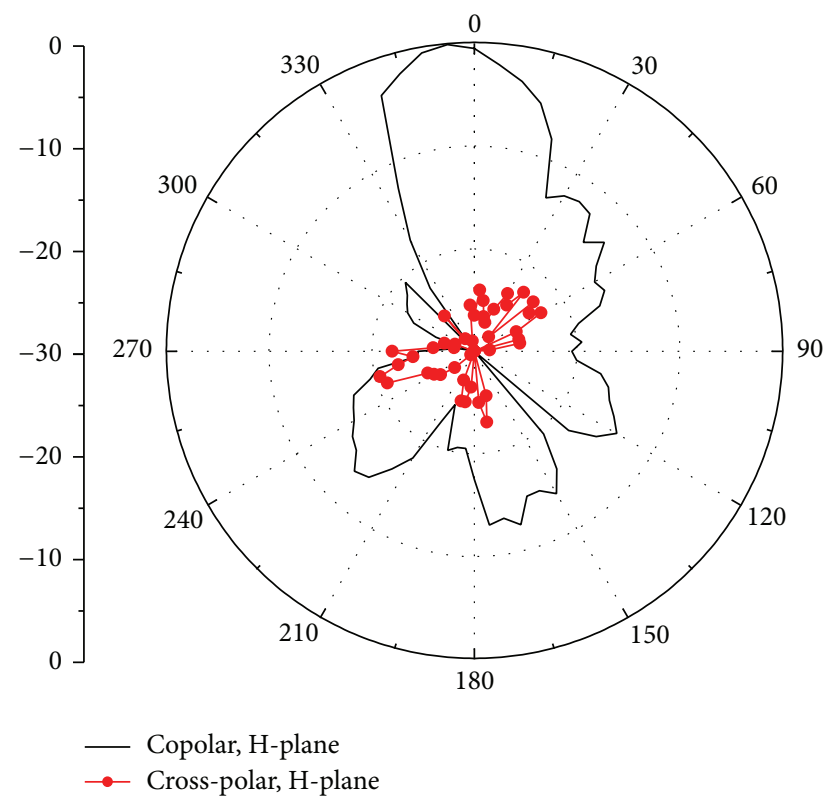

(d)

Figure 7: Measured radiation patterns at 9.8 GHz: (a) Port1 E-plane, (b) Port1 H-plane, (c) Port2 E-plane, and (d) Port2 H-plane.

in dual frequency bands. The measured cross-polarization, isolation, and operating bandwidth of two ports of these antennas are compared in Table 2. The result shows that the proposed antenna not only achieves low cross-polarization and high isolation but also widens the bandwidth, which is the major bottleneck of FPC antenna technology for wideband application.

\section{Conclusion}

A dual-polarization substrate-integrated Fabry-Pérot cavity antenna with near-field-coupled and slot-coupled feeding structures for different ports was presented in this paper. The symmetrical feeding structure of the proposed antenna contributes to purely linear polarization and high isolation $(>40 \mathrm{~dB})$. The antenna prototype is fabricated for verification and the measured results agree with the simulated results. The measured impedance bandwidth is larger than $8.3 \%$ $(9.44-10.26 \mathrm{GHz})$ for both ports and the maximum gain is $15.5 \mathrm{dBi}$ for $\mathrm{V}$-polarization and $14.9 \mathrm{dBi}$ for $\mathrm{H}$-polarization. The proposed antenna has a great potential for high gain and dual-polarization applications due to its mechanical robustness and simple structure.

\section{Conflict of Interests}

The authors declare that there is no conflict of interests regarding the publication of this paper. 


\section{Acknowledgment}

This work is supported by the Youth Innovation Foundation of Ministry of Education of China (no. WK2100060018).

\section{References}

[1] B. Lindmark and M. Nilsson, "On the available diversity gain from different dual-polarized antennas," IEEE Journal on Selected Areas in Communications, vol. 19, no. 2, pp. 287-294, 2001.

[2] C. T. P. Song, A. Mak, B. Wong, D. George, and R. D. Murch, "Compact low cost dual polarized adaptive planar phased array for WLAN," IEEE Transactions on Antennas and Propagation, vol. 53, no. 8, pp. 2406-2416, 2005.

[3] G. von Trentini, "Partially reflecting sheet arrays," IEEE Transactions on Antennas and Propagation, vol. 4, no. 4, pp. 666-671, 1956.

[4] H. Moghadas, M. Daneshmand, and P. Mousavi, "A dual-band high-gain resonant cavity antenna with orthogonal polarizations," IEEE Antennas and Wireless Propagation Letters, vol. 10, pp. 1220-1223, 2011.

[5] Z.-B. Weng, Y. Song, Y.-C. Jiao, and F.-S. Zhang, "A directive dual-band and dual-polarized antenna with zero index metamaterial," Microwave and Optical Technology Letters, vol. 50, no. 11, pp. 2902-2904, 2008.

[6] B. Zhu, Z. N. Chen, and Y. Feng, "Fully substrate-integrated high-gain thin Fabry-Perot cavity antennas," in Proceedings of the Asia-Pacific Microwave Conference, pp. 602-605, Melbourne, Australia, December 2011.

[7] L. Ji, J. Wang, W. Chen, and C. Chen, "Substrate-integrated Fabry-Pérot cavity antenna fed by slot-coupled patch array for directivity enhancement," in Proceedings of the 3rd AsiaPacific Microwave Conference (APMC '13), pp. 1067-1069, Seoul, Republic of Korea, November 2013.

[8] Y. Sun, Z. N. Chen, Y. Zhang, H. Chen, and T. S. P. See, "Subwavelength substrate-integrated Fabry-Pérot cavity antennas using artificial magnetic conductor," IEEE Transactions on Antennas and Propagation, vol. 60, no. 1, pp. 30-35, 2012.

[9] A. P. Feresidis, G. Goussetis, S. Wang, and J. C. Vardaxoglou, "Artificial magnetic conductor surfaces and their application to low-profile high-gain planar antennas," IEEE Transactions on Antennas and Propagation, vol. 53, no. 1, pp. 209-215, 2005.

[10] M. Barba, "A high-isolation, wideband and dual-linear polarization patch antenna," IEEE Transactions on Antennas and Propagation, vol. 56, no. 5, pp. 1472-1476, 2008. 

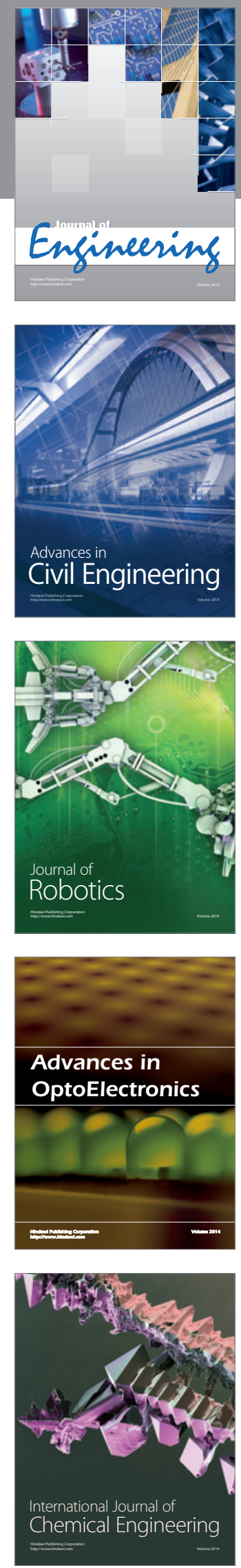

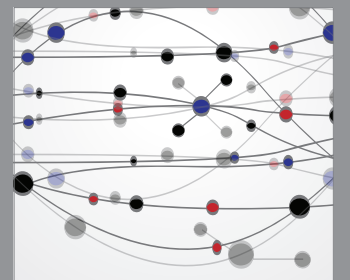

The Scientific World Journal
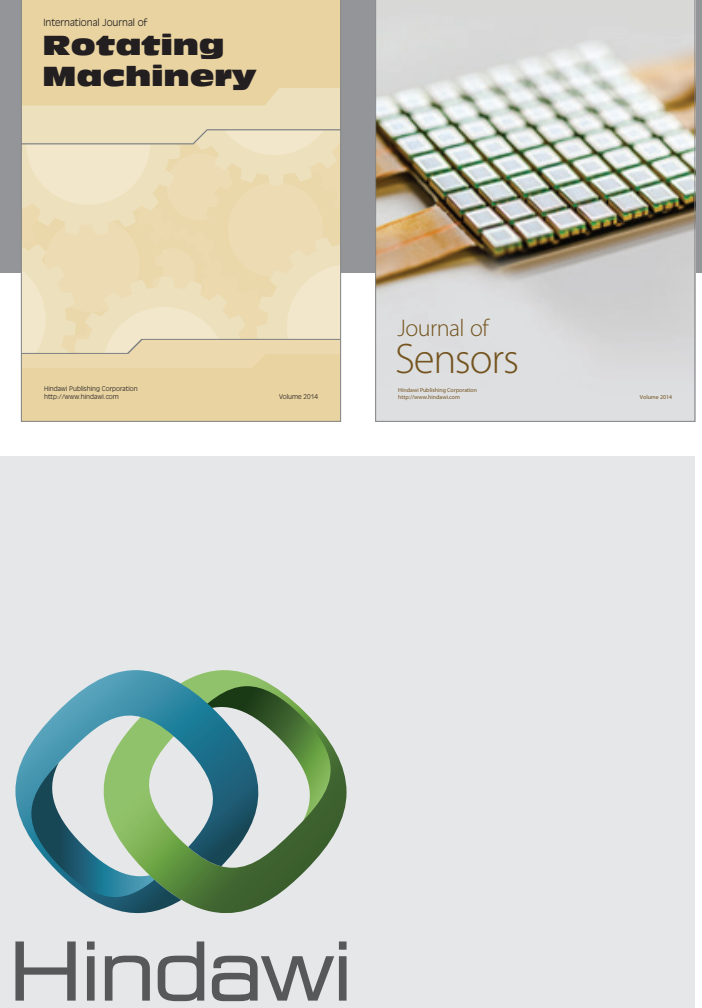

Submit your manuscripts at http://www.hindawi.com
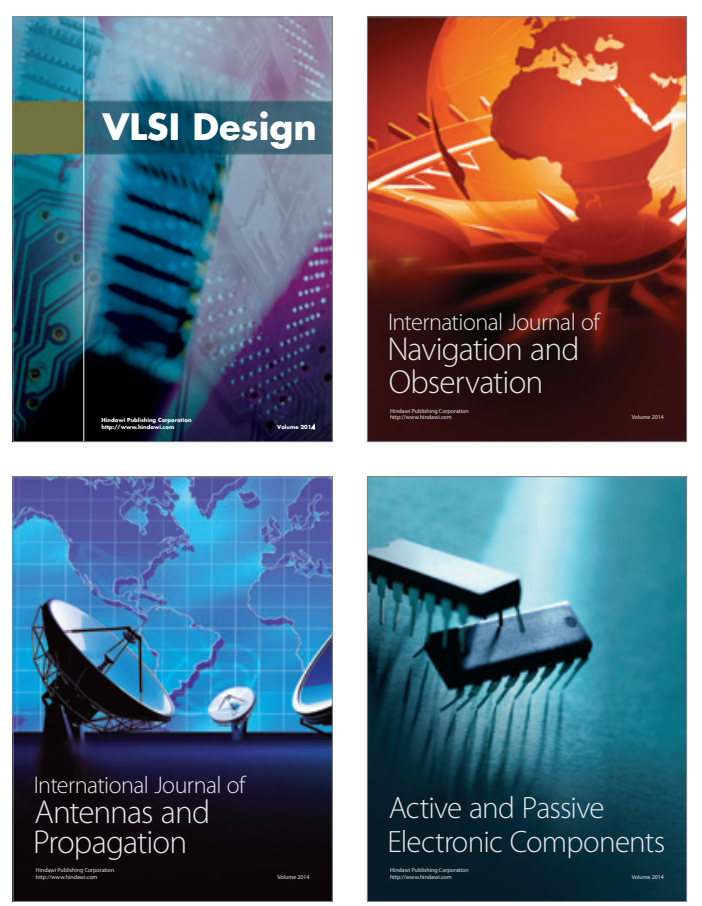
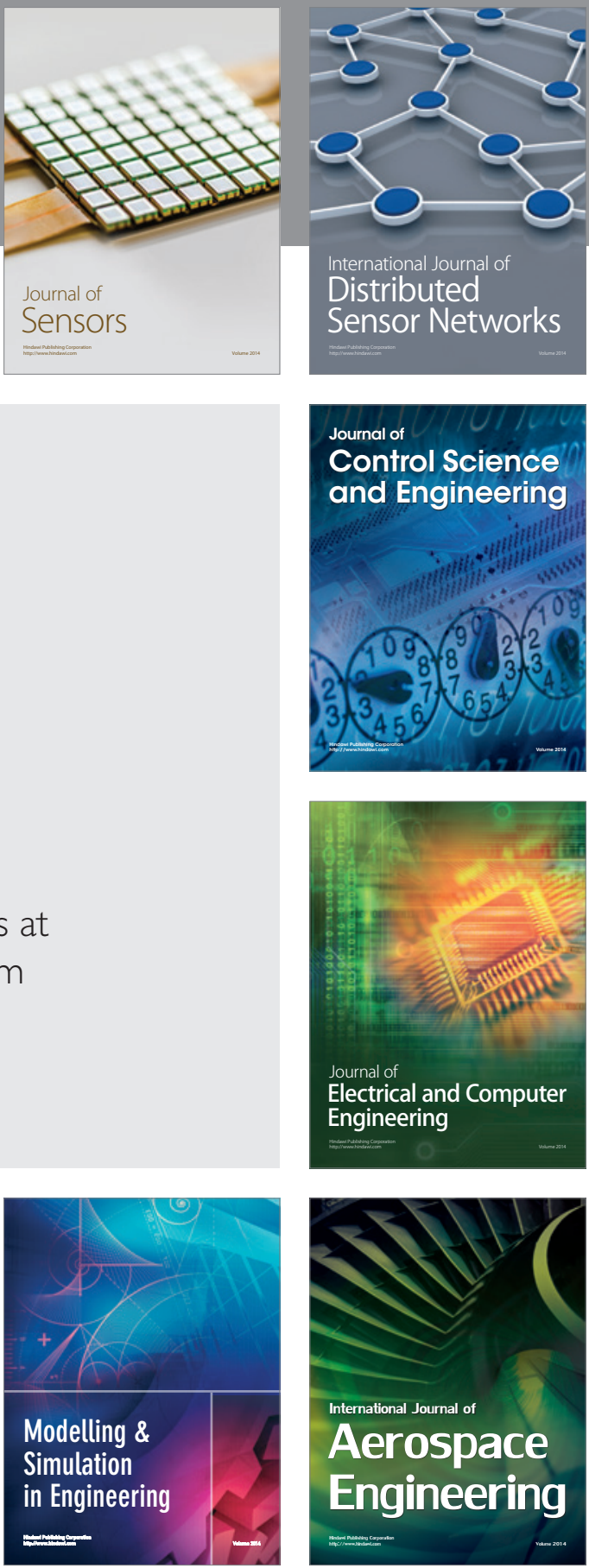

Journal of

Control Science

and Engineering
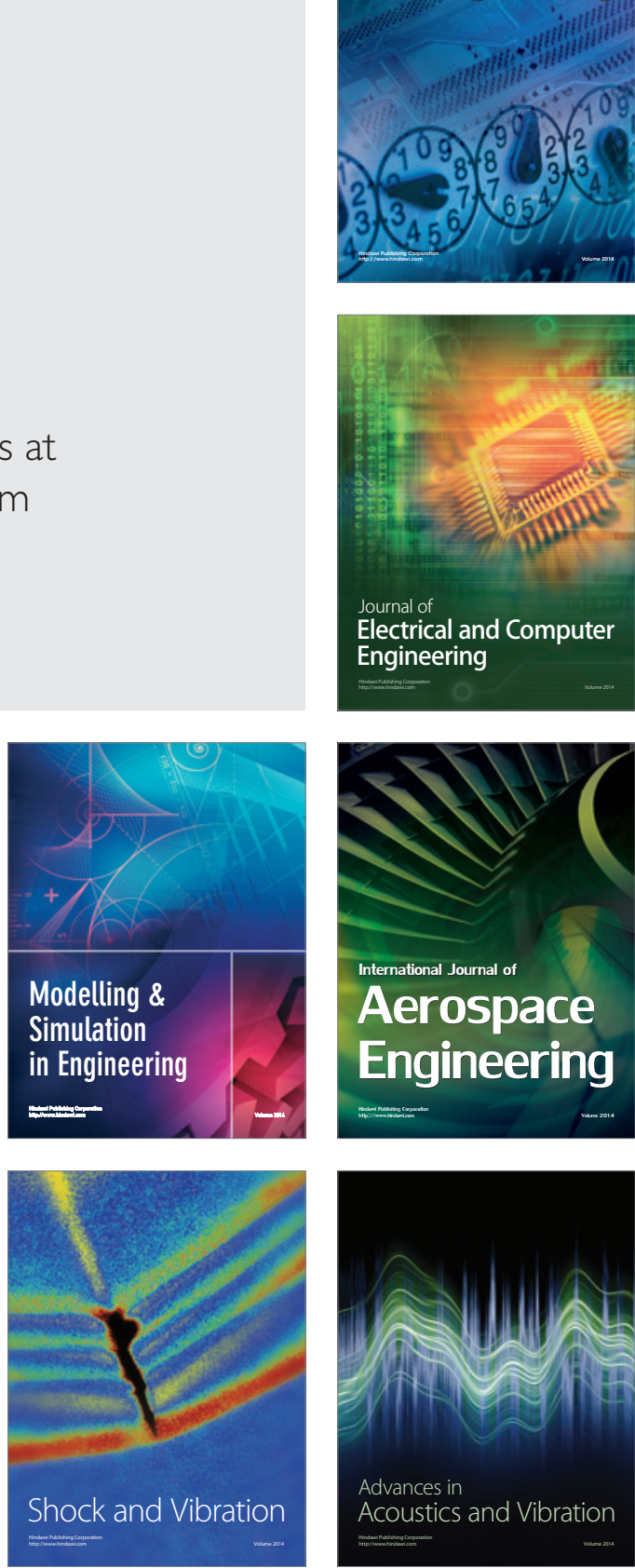